\title{
Magnetically induced electrostimulation of human osteoblasts results in enhanced cell viability and osteogenic differentiation
}

\author{
BETTINA HIEMER * , JOSEFIN ZIEBART* ${ }^{*}$ ANIKA JONITZ-HEINCKE, \\ PHILIP CHRISTIAN GRUNERT, YUKUN SU, DORIS HANSMANN and RAINER BADER \\ Department of Orthopaedics, Biomechanics and Implant Technology Research Laboratory, \\ University Medical Center Rostock, 18057 Rostock, Germany
}

Received January 21, 2016; Accepted April 14, 2016

DOI: $10.3892 / \mathrm{ijmm} .2016 .2590$

\begin{abstract}
The application of electromagnetic fields to support the bone-healing processes is a therapeutic approach for patients with musculoskeletal disorders. The ASNIS-III s-series screw is a bone stimulation system providing electromagnetic stimulation; however, its influence on human osteoblasts (hOBs) has not been extensively investigated. Therefore, in the present study, the impact of this system on the viability and differentiation of hOBs was examined. We used the ASNIS-III s screw system in terms of a specific experimental test set-up. The ASNIS-III s screw system was used for the application of electromagnetic fields (EMF, $3 \mathrm{mT}, 20 \mathrm{~Hz}$ ) and electromagnetic fields combined with an additional alternating electric field (EMF + EF) $(3 \mathrm{mT}, 20 \mathrm{~Hz}, 700 \mathrm{mV})$. The stimulation of primary hOBs was conducted 3 times per day for 45 min over a period of $72 \mathrm{~h}$. Unstimulated cells served as the controls. Subsequently, the viability, the gene expression of differentiation markers and pro-collagen type 1 synthesis of the stimulated osteoblasts and corresponding controls were investigated. The application of both EMF and EMF + EF using the ASNIS-III s screw system revealed a positive influence on bone cell viability and moderately increased the synthesis of pro-collagen type 1 compared to the unstimulated controls. Stimulation with EMF resulted in a slightly enhanced gene expression of type 1 collagen and osteocalcin; however, stimulation with EMF + EF resulted in a significant increase in alkaline phosphatase (1.4-fold) and osteocalcin (1.6-fold) levels, and a notable increase in the levels of runt-related transcription factor 2 (RUNX-2; 1.54-fold). Our findings demonstrate that stimulation with electromagnetic fields and an additional alternating electric field has a positive
\end{abstract}

Correspondence to: Ms. Bettina Hiemer, Department of Orthopaedics, Biomechanics and Implant Technology Research Laboratory, University Medical Center Rostock, Doberaner Strasse 142, Rostock 18057, Germany

E-mail: bettina.hiemer@med.uni-rostock.de

\section{${ }^{*}$ Contributed equally}

Key words: bone tissue, electromagnetic stimulation, electric field, osteoblasts, regeneration influence on hOBs as regards cell viability and the expression of osteoblastic differentiation markers.

\section{Introduction}

Bone tissue is subjected to constant remodelling processes mediated by bone-forming osteoblasts and bone-resorbing osteoclasts. According to Wolff's law, these processes are not random, but are a consequence of bone tissue responding to mechanical stress (1). Fukada and Yasuda demonstrated the piezoelectric properties of bone and showed that mechanical stress induces the formation of endogenous electric fields within the tissue (2). Several studies have been conducted to investigate whether bone healing can be influenced by electrical stimulation. Although the underlying mechanisms of electrically induced osteogenesis are not yet completely understood, previous findings have demonstrated the benefit of applying electromagnetic fields on bone regeneration. An in vitro study by Icaro Cornaglia et al using the human osteosarcoma cell line, SAOS-2, showed significantly increased matrix calcification following stimulation with electromagnetic fields (3). In other studies, an association between the use of low-frequency electromagnetic fields and enhanced collagen synthesis in mouse osteoblasts was observed (4). Electric $(5,6)$ and electromagnetic (7-9) fields even support the differentiation of human mesenchymal stem cells into the osteoblastic phenotype. These effects are mediated through direct effects on intracellular and transmembrane channels (10), as well as through indirect effects through the inverse piezoelectric effect $(11,12)$.

It has also been shown in vitro and in vivo, that biophysical stimulation via the application of electric currents enhances bone healing and restores structural strength (13-15). Based on these findings, electrical bone growth stimulators have been developed for clinical application $(16,17)$. These systems provide external stimulation that imitates endogenous electric fields in order to activate bone regeneration. Common bone stimulator techniques represent a promising therapeutic approach for diseases, such as osteoarthritis and osteoporosis, as well as for complicated fractures, including delayed unions, non-unions and stress fractures (18). A particular technique for the application of electromagnetic fields with an additional alternating electric field (EMF + EF) is the ASNIS-III s screw 
system (Stryker GmbH, Duisburg, Germany). This system is based on the bipolar induction screw system (BISS) (19) that is derived from the stimulation method developed by Mittelmeier et al. This semi-invasive method has been described in detail previously $(12,19)$. The ASNIS screw is implanted at the site of the bone defect. A magnetic field of 3-5 $\mathrm{mT}$ oscillating at a $20 \mathrm{~Hz}$ sine wave, generated by an external primary coil induces voltage within the secondary coil inside the ASNIS screw. This secondary coil is connected to two electrodes in the screw tip and shaft, which are separated by electrical insulation. As a result, an electric field is created between the two electrodes. The maximum root mean square (RMS) electric potential on the surface of the electrodes is $700 \mathrm{mV}$ (Fig. 1).

The ASNIS-III s screw system is a bone-stimulating implant that is already being applied in clinical practice for the treatment of avascular necrosis of the femoral head, fracture of the femur neck and subtalar arthrodeses. The ASNIS-III s screw system applies electromagnetic fields and an additional electric field by a single screw and directly stimulates the adjacent bone tissue which is supposed to accelerate bone regeneration. Although the ASNIS-III s system is already being used clinically, optimal parameters of stimulation (electric field strength, frequency and stimulation periods) to further enhance the effects of electromagnetic stimulation are still unknown. Therefore, we developped a three-dimensional (3D) in vitro test set-up using the technical equipment of the ASNIS-III s screw system (12). In this set-up, the influence of EMF, as well as $\mathrm{EMF}+\mathrm{EF}$ on bone cells on different biomaterials was investigated. This in vitro study showed an early shift of the osteoblasts towards differentiation after a stimulation period of 3 days when seeded on collagen scaffolds, indicating the influence of piezoelectric materials on the stimulatory effects. However, several studies stimulating cells on non-piezoelectric materials have also demonstrated the effects of electromagnetic stimulation (20-22). Therefore, in the present study, we focused on the electromagnetic stimulation of human osteoblasts (hOBs) in the absence of a matrix displaying piezoelectric properties to reveal the direct effect of electromagnetic stimulation on cells. Hence, in the present study, hOBs were integrated in agarose gels enabling the stimulation of osteoblasts in a 3D matrix and facilitating RNA isolation for subsequent gene expression analysis. In such a set-up, the influence of EMF and EMF + EF on the viability and differentiation capacity of primary hOBs was analysed.

\section{Materials and methods}

Isolation of hOBs and embedding in agarose scaffold.Primary hOBs were isolated under sterile conditions from the femoral heads of patients undergoing a primary total hip replacement as previously described (23). The samples were collected with the consent of patients and after approval by the local ethics committee (registration number: A 2010-10).

Isolated cells were cultured in $25 \mathrm{~cm}^{2}$ flasks with $8 \mathrm{ml}$ of Dulbecco's modified Eagle's medium (Biochrom, Berlin, Germany) containing $10 \%$ fetal calf serum (FCS), $1 \%$ amphotericin $\mathrm{B}, 1 \%$ penicillin-streptomycin and $1 \%$ HEPES buffer under standard cell culture conditions $\left(5 \% \mathrm{CO}_{2}\right.$ and $\left.37^{\circ} \mathrm{C}\right)$. Osteogenic differentiation was induced by ascorbic

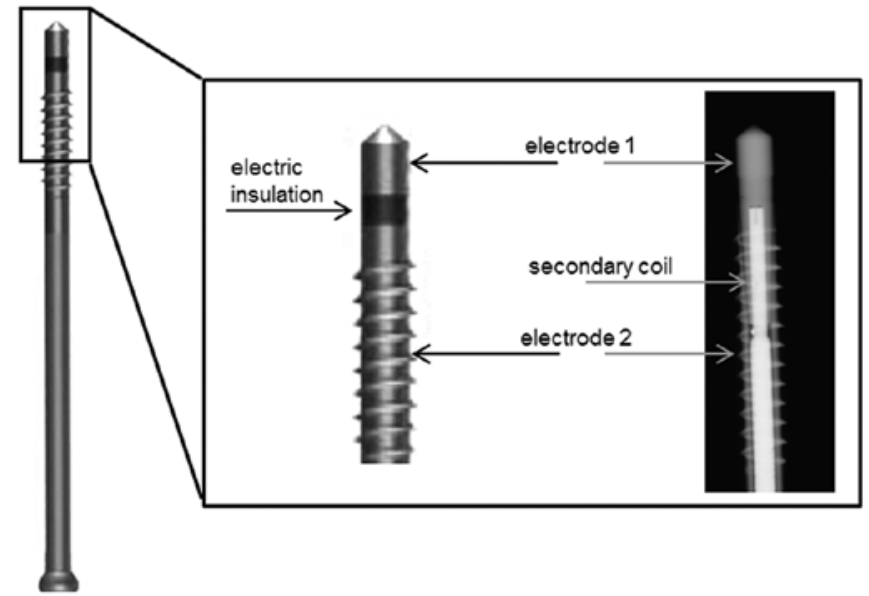

Figure 1. ASNS-III s screw used for electromagnetic fields combined with an additional alternating electric field (EMF + EF) stimulation of bone tissue. A secondary coil is integrated into a single screw (X-ray image) and is connected to two electrodes in the shaft and tip. An electrical insulation separates the two electrodes [modified from Grunert et al (12)].

acid $(50 \mu \mathrm{g} / \mathrm{ml}), \beta$-glycerophosphate $(10 \mathrm{mM})$ and dexamethasone $(100 \mathrm{nM})$, and verified by the immunhistochemical detection of the enzyme alkaline phosphatase (ALP) using the Fuchsin+ Substrate Chromogen System (Dako, Hamburg, Germany) according to the manufacturer's instructions.

At passage 3 the hOBs were embedded in an agarose scaffold using low gelling agarose (Sigma-Aldrich, Seelze, Germany). The solid agarose was dissolved with distilled water and sterilised at $135^{\circ} \mathrm{C}$ for $1 \mathrm{~h}$. After cooling down for $24 \mathrm{~h}$ at $37^{\circ} \mathrm{C}$, the agarose solution was diluted with cell culture medium, resulting in a $1 \%$ agarose solution.

The cultured cells were detached with trypsin/EDTA solution and centrifuged to a pellet at $118 \mathrm{x} \mathrm{g}$. Subsequently, $8.25 \times 10^{5}$ cells were resuspended in $3 \mathrm{ml}$ liquid $1 \%$ agarose solution and transferred into inserts for 6-well cell culture plates (ThinCert ${ }^{\mathrm{TM}}$; Greiner Bio-One, Frickenhausen, Germany). Cell-agarose solution was gelled at $4^{\circ} \mathrm{C}$ for $3 \mathrm{~min}$. Afterwards, an agarose scaffold measuring $30 \mathrm{~mm}$ in diameter and $5 \mathrm{~mm}$ in height was prepared. The centre of the cell-agarose scaffold was cut out in order to position the ASNIS screw in the middle of the scaffold. The stimulation of $7.5 \times 10^{5}$ hOBs required the use of $10 \%$ cell excess to compensate the loss of scaffold during the preparation of the experimental set-up.

Experimental set-up for magnetic and electromagnetic stimulation. EMF and EMF + EF were applied in vitro using the ASNIS-III s-series screw system (Stryker GmbH and Co. KG, Duisburg, Germany) as previously described (12). Custom-made polystyrole screw holders with a diameter of $3.5 \mathrm{~cm}$ were placed in 6-well cell culture plates to enable the stable alignment of the ASNIS screw. The screw holder was covered by the agarose scaffold and the construct was overlaid with $6 \mathrm{ml}$ cell culture medium containing supplements for osteogenic differentiation as mentioned above. The ASNIS screw was adjusted within the centre of the agarose scaffold and screw holder by screwing them through a pre-drilled hole in the lid of the 6-well plate (Fig. 2A-E). The cells were incubated for $24 \mathrm{~h}$ to ensure cell adaption within the scaffold. 

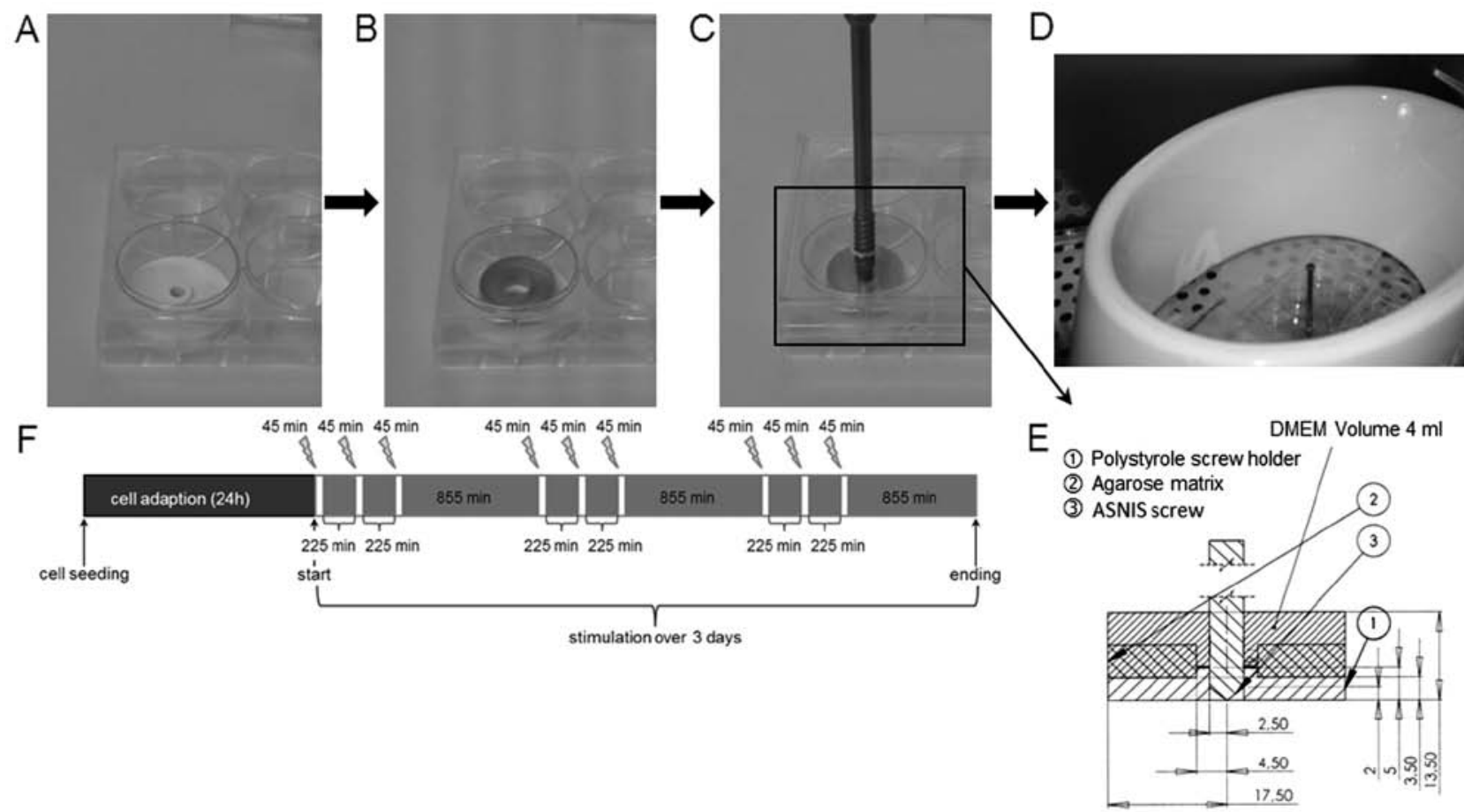

Figure 2. (A-E) Experimental set-up. In vitro test system for (B) EMF and (C) electromagnetic fields combined with an additional alternating electric field (EMF + EF) stimulation using the ASNIS-III s screw system. (F) Flow chart of stimulation protocol [modified from Grunert et al (12)].

EMF stimulation was performed by positioning the cell culture plate with agarose-cell scaffolds and custom screw holders (Fig. 2B) within the primary magnetic coil. The coil generated a sinusoidal oscillating magnetic field with a frequency of $20 \mathrm{~Hz}$ and a magnetic flux density of $3 \mathrm{mT}$. The addition of the ASNIS screw (Fig. 2C) enabled the application of EMF + EF on the hOBs. The maximum induced voltage between the electrodes of the ASNIS screw was $700 \mathrm{mV}$, as previously described (12). The cells were stimulated (EMF and $\mathrm{EMF}+\mathrm{EF}) 3$ times per day for 45 min over a period of 3 days (Fig. 2F). The cell culture conditions were $37^{\circ} \mathrm{C}$ and $5 \% \mathrm{CO}_{2}$ in a humidified atmosphere. Unstimulated cells embedded in an agarose scaffold with (EMF + EF control) and without (EMF control) the ASNIS screw served as the controls and were located in a separate incubator to avoid influences from the magnetic field generated by the external magnetic coil.

Determination of cell viability. The metabolic activity of the cells was examined using the water-soluble tetrazolium salt (WST)-1 assay (Roche, Berlin, Germany). This colorimetric assay is based on the reduction of water soluble tetrazolium salt into formazan salt catalyzed by mitochondrial dehydrogenases in intact cells. The amount of formazan correlates with the enzymatic activity and reflects the metabolic activity. Following incubation with a mix of the WST assay reagent and cell culture medium at a ratio of 1:10 for $120 \mathrm{~min}$ at $37^{\circ} \mathrm{C}$, the absorbance was measured at $450 \mathrm{~nm}$ (reference, $630 \mathrm{~nm}$ ) using an Opsys MR microplate reader (Dynex Technologies, Denkendorf, Germany).

The viability of the osteoblasts was assessed using a LIVE/DEAD ${ }^{\odot}$ assay kit (Life Technologies, Carlsbad, CA, USA). The two-color assay discriminates live from dead cells by simultaneously staining with green fluorescent (494-517 nm) calcein-acetoxymethyl (calcein-AM), indicating intracellular esterase activity and red-fluorescent $(528-617 \mathrm{~nm})$ ethidium homodimer-1, indicating the loss of plasma membrane integrity. The assay was performed as recommended by the manufacturer. Images of the cells were acquired using a fluorescence microscope (Nikon Type 120) and evaluated with NIS-Elements software (version D 3.2) (both from Nikon Instruments, Tokyo, Japan). Separate images of live and dead cells were taken in the same position. Afterwards, the images were overlaid using image editor software (GIMP2.8.14, The GIMP Team).

Gene expression analysis. After a stimulation period of 3 days, all samples were frozen at $-70^{\circ} \mathrm{C}$ for subsequent gene expression analysis. The samples were thus quickly covered with liquid nitrogen and homogenised using a pre-cooled pestle and mortar. The fine powder was transferred into a centrifuge tube; subsequently, TRI Reagent ${ }^{\circledR}$ (Zymo Research, Freiburg, Germany) and Buffer QG (Qiagen, Leipzig, Germany) were added. After mixing and incubating at room temperature for $5 \mathrm{~min}$, the samples were centrifuged for $2 \mathrm{~min}$ at $12000 \mathrm{xg}$. The supernatants were used to perform RNA isolation by column purification using the Direct-zol ${ }^{\mathrm{TM}}$ RNA MiniPrep kit (Zymo Research) according to the manufacturer's instructions.

Single-stranded cDNA was synthesised from total RNA with a T-Personal Thermocycler (Biometra, Göttingen, Germany) using the High Capacity cDNA reverse transcription kit following the manufacturer's instructions (Applied Biosystems, Forster City, CA, USA). The synthesised cDNA was used as a template for semi-quantitative real-time polymerase chain reaction (RT-qPCR) using the innuMIX qPCR MasterMix SyGreen and qTower 2.0 (Analytik Jena AG, Jena, Germany). The cycling conditions used for amplification were $95^{\circ} \mathrm{C}$ for $2 \mathrm{~min}, 40$ cycles of $95^{\circ} \mathrm{C}$ for $5 \mathrm{sec}$ and $65^{\circ} \mathrm{C}$ for $25 \mathrm{sec}$. 
Table I. Sequences of primers used for RT-qPCR.

\begin{tabular}{|c|c|c|}
\hline Gene & Direction & Primer nucleotide sequence \\
\hline Alkaline phosphatase $(A L P)$ & $\begin{array}{l}\text { Forward } \\
\text { Reverse }\end{array}$ & $\begin{array}{l}\text { 5'-CATTGTGACCACCACGAGAG-3' } \\
\text { 5'-CCATGATCACGTCAATGTCC-3' }\end{array}$ \\
\hline Bone sialoprotein $(B S P)$ & $\begin{array}{l}\text { Forward } \\
\text { Reverse }\end{array}$ & $\begin{array}{l}\text { 5'-ATTTTGGGAATGGCCTGTGC-3' } \\
\text { 5'-GTCACTACTGCCCTGAACTGG-3' }\end{array}$ \\
\hline Collagen type 1 (CollAl) & $\begin{array}{l}\text { Forward } \\
\text { Reverse }\end{array}$ & $\begin{array}{l}\text { 5'-ACGAAGACATCCCACCAATC-3' } \\
\text { 5'- AGATCACGTCATCGCACAAC-3' }\end{array}$ \\
\hline Hypoxanthine-guanine phosphoribosyltransferase (HPRT) & $\begin{array}{l}\text { Forward } \\
\text { Reverse }\end{array}$ & $\begin{array}{l}\text { 5'-CCCTGGCGTCGTGATTAGTG-3' } \\
\text { 5'-TCGAGCAAGACGTTCAGTCC-3' }\end{array}$ \\
\hline Osteocalcin $(O C)$ & $\begin{array}{l}\text { Forward } \\
\text { Reverse }\end{array}$ & $\begin{array}{l}\text { 5'-GGTGCAGCCTTTGTGTCC-3' } \\
\text { 5'-TCAGCCAACTCGTCACAGTC-3' }\end{array}$ \\
\hline Runt-related transcription factor $2(R U N X-2)$ & $\begin{array}{l}\text { Forward } \\
\text { Reverse }\end{array}$ & $\begin{array}{l}\text { 5'-CGCCTCACAAACAACCACAG-3' } \\
\text { 5'-ACTGCTTGCAGCCTTAAATGAC-3' }\end{array}$ \\
\hline
\end{tabular}

The sequences of the forward and reverse primers are shown in Table I. The expression of all genes was normalised to the expression of the corresponding housekeeping gene, hypoxanthine phosphoribosyltransferase 1 (HPRT). The relative amount of target mRNA in thye unstimulated cells and treated cells was analysed using the $\Delta \Delta \mathrm{Cq}$ method, where $\Delta \Delta \mathrm{Cq}=\Delta \mathrm{Cq}_{\text {stimulaion }^{-}}$ $\Delta \mathrm{Cq}_{\text {control }}$, as previously described (24).

Determination of pro-collagen type I protein content. The rate of synthesis of the pro-collagen type I rate was measured using an enzyme-linked immunosorbent assay (ELISA) (MicroVue $^{\mathrm{TM}}$ CICP EIA; Quidel Corporation, San Diego, USA). The C-terminal pro-peptide of pro-collagen is considered to correlate with collagen expression. For the analysis, supernatants of each stimulation experiment were collected and stored at $-20^{\circ} \mathrm{C}$. The assay was performed according to the manufacturer's specifications. The absorbance was measured at a wavelength of $405 \mathrm{~nm}$ using an Opsys MR microplate reader (Dynex Technologies). With the help of a standard curve, the protein content was determined and set in relation to the respective controls.

Data illustration and statistical analysis. Data are represented in a box plot. Each box shows the median, as well as the 25 th and 75 th percentile. Dots point out mean values. The whiskers indicate the minimum and maximum data values. A minimum of 4 independent experiments were performed for statistical analysis. The Kolmogorov-Smirnov-test was conducted to assess the distribution of data. As the data sets were found to be distributed normally, the statistical significance of differences between groups was calculated by one-way ANOVA (with the Bonferroni post hoc test) using SPSS Statistics 2.0 software (IBM, Ehningen, Germany). The level of significance was set to $\mathrm{p}<0.05$.

\section{Results}

Viability of hOBs following stimulation with EMF and $E M F+E F$. In this study, we examined the influence of EMF and EMF + EF on the survival and differentiation of hOBs using the ASNIS-III s screw system. Using the WST assay, the metabolic activity of the hOBs was detected and represented in relation to the respective unstimulated controls (Fig. 3A). Accordingly, EMF + EF (1.24-fold) and EMF (1.27-fold) increased the metabolic activity of the hOBs compared to the unstimulated cells. Furthermore, the viability of the hOBs was detected by live/dead staining. Osteoblasts stimulated with $\mathrm{EMF}$ and $\mathrm{EMF}+\mathrm{EF}$ as well as the respective unstimulated controls displayed a large number of green fluorescent, viable cells. Additionally, dead cells (red fluorescence) were observed in each stimulation group (Fig. 3B). No differences in the proportion of living and dead cells were detected between $\mathrm{EMF}, \mathrm{EMF}+\mathrm{EF}$ and the controls.

Alteration of osteogenic differentiation in hOBs induced by $E M F$ and $E M F+E F$. Gene expression analyses of commonly used osteogenic markers were performed to investigate the differentiation of hOBs following stimulation with EMF and $\mathrm{EMF}+\mathrm{EF}$. During exposure to EMF, the expression of collagen type 1 (CollAl; 1.21-fold) and osteocalcin $(O C ; 1.31$-fold) slightly increased compared to the corresponding unstimulated controls, whereas the expression of runt-related transcription factor 2 (RUNX-2; 0.93-fold) and bone sialoprotein (BSP; 0.84-fold) was slightly downregulated. However, stimulation with EMF $+\mathrm{EF}$ resulted in a significant increase in the expression of ALP compared to both EMF ( $\mathrm{p}=0.048)$ and the unstimulated control (1.4-fold, $\mathrm{p}=0.036)$. Moreover, $O C$ expression was significantly enhanced following stimulation with EMF + EF (1.6-fold, p=0.017) compared to the unstimulated cells. The transcription factor, $R U N X-2$, exhibited an increased expression level (1.5-fold), while CollAl was only marginally influenced (Fig. 4).

Effect of EMF and EMF + EF on the synthesis of C1CP. Regarding the synthesis capacity of specific extracellular matrix components, we determined the content of collagen type 1 using the pro-collagen type 1 (C1CP) ELISA. The $\mathrm{C} 1 \mathrm{CP}$ concentrations of EMF, EMF $+\mathrm{EF}$ and the respective 
A

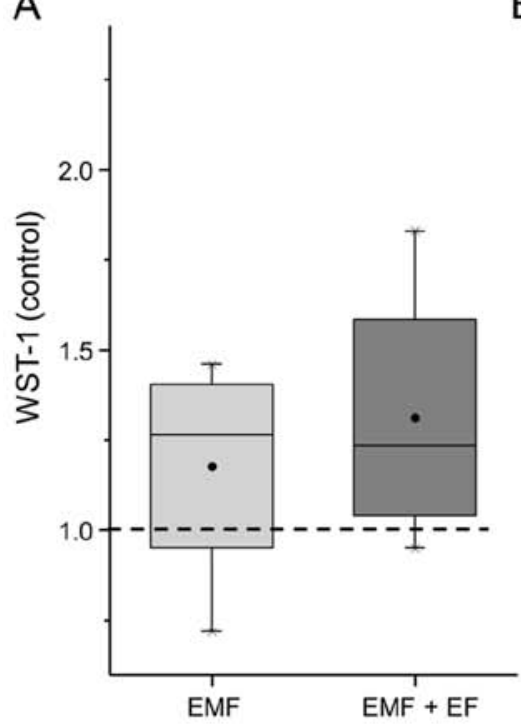

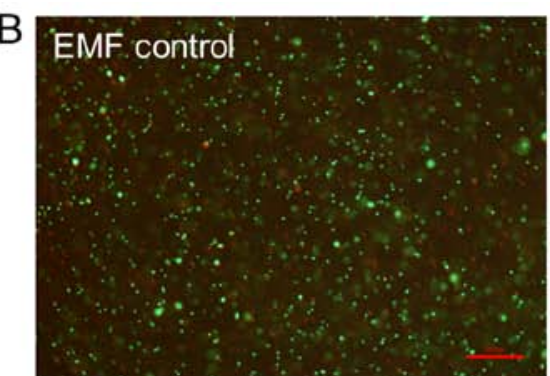
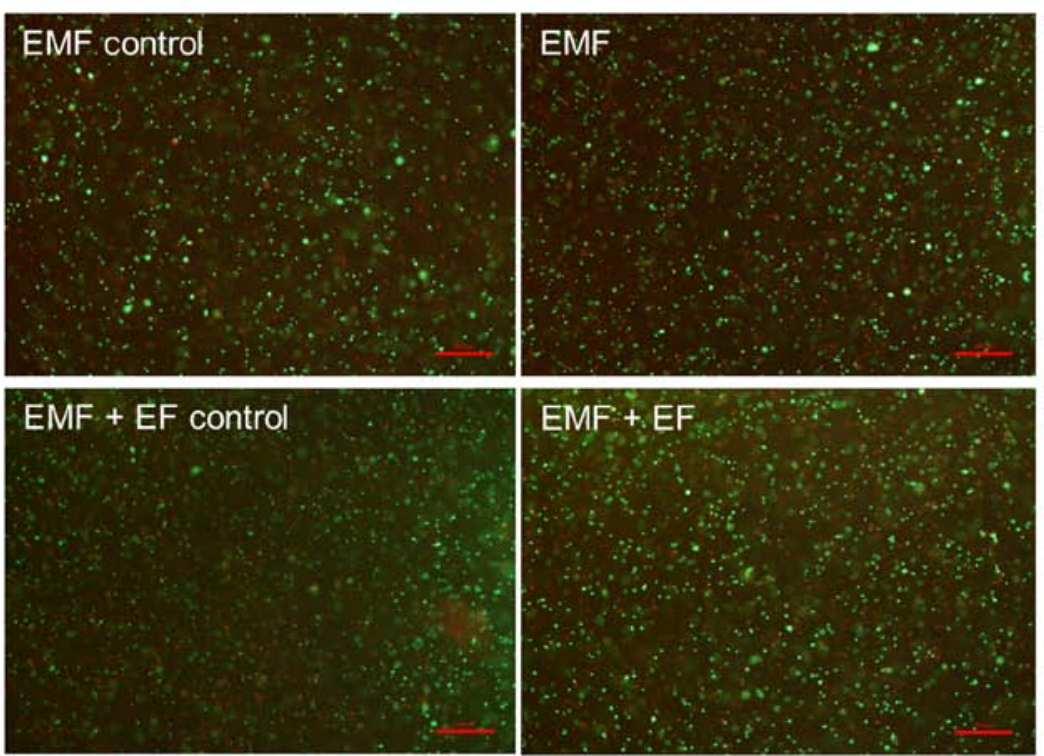

Figure 3. Influence of EMF and electromagnetic fields combined with an additional alternating electric field (EMF + EF) on metabolic activity and viability of human osteoblasts after 3 days. (A) Metabolic activity was quantified using WST-1 assay. Data (n=4) are normalised to the respective control and presented in box plots, whereby boxes identify interquartile ranges, horizontal lines within the boxes indicate the median, black dots show the mean and whiskers denote minimum and maximum values. Statistical analysis was conducted by one-way ANOVA (p<0.05). (B) Fluorescent microscopic photographs of live/dead staining. Viable cells fluoresced green and dead cells fluoresced red (bar, $200 \mu \mathrm{m}$ ).

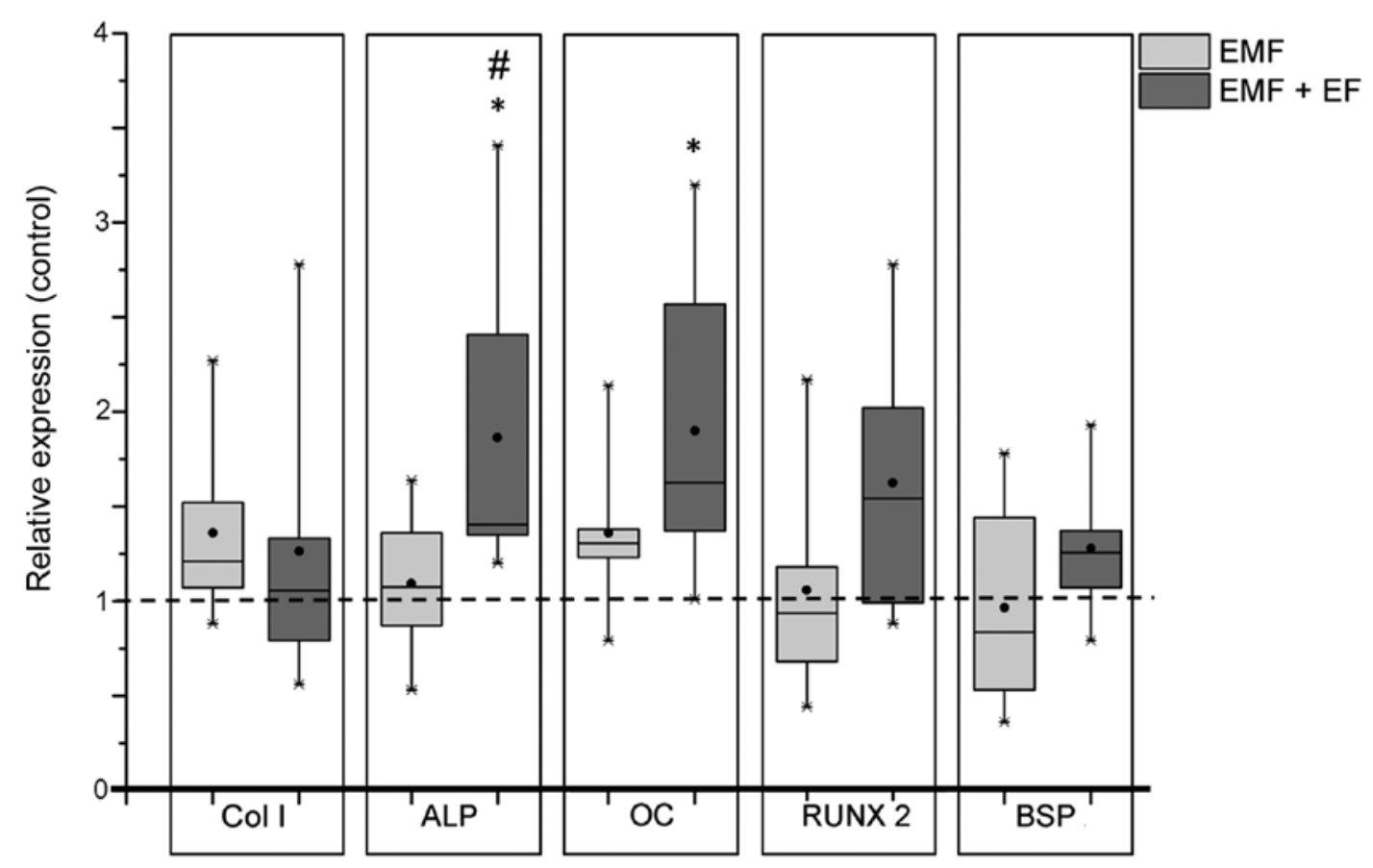

Figure 4. Influence of EMF and electromagnetic fields combined with an additional alternating electric field (EMF + EF) stimulation on gene expression of human osteoblasts after 3 days. Gene expression levels of osteogenic markers after EMF and EMF + EF in relation to the non-treated control. Data ( $\mathrm{n}=6$ ) are presented in box plots, whereby boxes identify interquartile ranges, horizontal lines within the boxes indicate the median, black dots show the mean and whiskers denote minimum and maximum values. Statistical analysis was conducted by one-way ANOVA ("p<0.05, represents significance of differences between EMF + EF and the controls; ${ }^{\#} \mathrm{p}<0.05$, represents significance of differences between EMF and EMF + EF). Target genes are collagen type 1 (Col I), alkaline phosphatase (ALP), osteocalcin (OC), runt-related transcription factor 2 (RUNX-2) and bone sialoprotein (BSP).

controls were measured after 3 days in the supernatant of the cells. The C1CP content of each stimulation group was set in relation to the corresponding control. C1CP expression was enhanced to a slightly greater extent by EMF (1.21-fold) than $\mathrm{EMF}+\mathrm{EF}$ (1.18-fold) (Fig. 5).

\section{Discussion}

Bone tissue has the ability to regenerate after lesions. This regeneration process is complex and bone regeneration of large defects after trauma, tumour development and avascular 


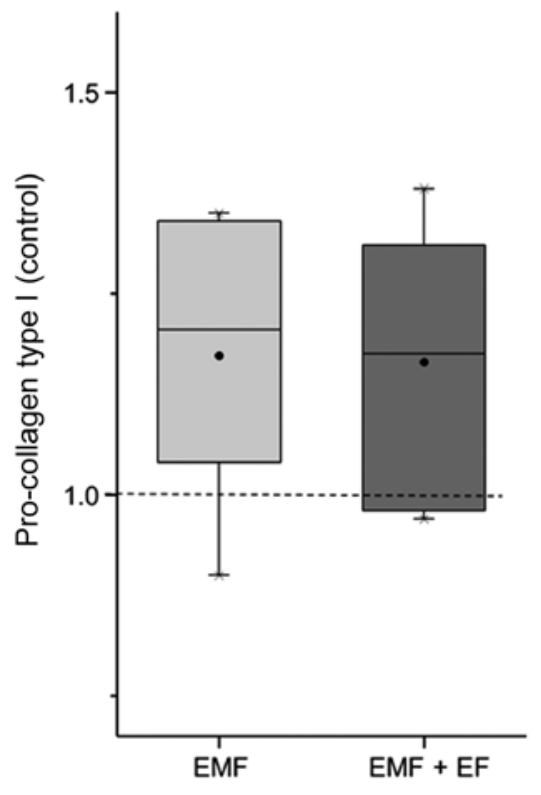

Figure 5. Influence of EMF and electromagnetic fields combined with an additional alternating electric field (EMF + EF) stimulation on the expression of pro-collagen type 1 in human osteoblasts after 3 days. Measurement of procollagen type 1 was performed using an ELISA test $(n=6)$. Data are normalised to the respective control and presented in box plots, whereby boxes identify interquartile ranges, horizontal lines within the boxes indicate the median, black dots show the mean and whiskers denote minimum and maximum values. Statistical analysis was conducted by one-way ANOVA $(p<0.05)$.

necrosis is one of the key challenges in reconstructive bone surgery (25). Current clinical approaches for the enhancement of bone regeneration are diverse; however, the use of bone grafting methods is considered the 'gold standard'. As these common treatments do not always achieve the success desired, the application of biophysical stimulation in combination with bone grafting is a promising therapeutic approach (9). Hence, the ASNIS-III s-series screw system, an electro-inductive bone stimulating system, was introduced to enhance bone regeneration in the case of avascular necrosis of the femoral head (19).

In a previous study, we established an in vitro test set-up for the stimulation of hOBs growing on 3D scaffolds made of collagen and calcium phosphate using the ASNIS-III s-series screw system (12). In the present study, we used this test set-up to investigate the influence of EMF and EMF + EF on the osteogenic differentiation of primary hOBs in the absence of extracellular matrix molecules. Therefore, we embedded hOBs in agarose gels to investigate the direct effects of electromagnetic stimulation. Agarose is highly biocompatible and its fibrous structure provides high porosity and similarity to physiological extracellular matrix (26). The suitability of agarose gels for the culture and differentiation of osteoblasts has been previously demonstrated for the mouse osteoblast cell line, MC3T3-E1 (27). Other groups have successfully implanted hydroxyapatite/agarose hydrogels as bone graft material, proving osteoconductive properties of composites $(26,28)$. Furthermore, the osteogenic differentiation of dental stromal cells under mechanical stimulation in agarose gels has been recently described (29). The ASNIS-III s screw was set in the centre of the 3D osteoblast-agarose scaffold to expose the bone cells to the EMF + EF. This set-up has the benefit of mimicking the situation in vivo, where the ASNIS-III s screw is implanted in the bone tissue to stimulate bone regeneration.

The ASNIS-III s treatment protocol for patients with avascular necrosis of the femoral head involves bone stimulation for $45 \mathrm{~min} 3$ times per day for several months. For our in vitro experiments, similar stimulation intervals over a stimulation period of 3 days were used to analyse the initial effects of electromagnetic stimulation, as done in a previous study by Grunert et al (12). In this study, no cytotoxic effects of the stimulation method or the screw material were determined. We approved the absence of the negative influence of the implant system, as there was no increase in cell death (assessed by live/dead staining) using the ASNIS screw compared to set-ups without the ASNIS screw. This confirms the biocompatibility of the Ti6Al4V alloy used for the electrodes (30-32) and rules out any negative effect of both EMF and EMF + EF. The exclusion of cytotoxic effects is a notable aspect, given the clinical application.

Our investigations indicate that hOBs cultured in agarose are sensitive to both EMF and EMF + EF, as for both conditions, metabolic activity was similarly enhanced compared to the unstimulated controls. In this study, cell survival and proliferation, assessed by live/dead staining, were comparable in all samples. However, other research groups have shown that EMF positively affects the proliferation of primary osteoblasts and osteoblast-like cell lines in non-piezoelectric scaffolds $(20,33)$.

Therapeutic success depends, not only on proliferation and cell viability, but also on the differentiation of osteoblasts with enhanced synthesis of extracellular matrix components required for regeneration processes. The osseous extracellular matrix is mainly composed of collagen type 1 . The analysis of the synthesised collagen type 1 levels showed a moderate increase following exposure to EMF and EMF + EF. Furthermore, gene expression analyses of important osteogenic differentiation markers were performed. Boxplots depicting gene expression demonstrate the variability of osteoblast donor susceptibility to electromagnetic stimulation which has to be taken into account for the evaluation of stimulatory effects and the potential outcome of EMF therapy. The stimulation of hOBs with EMF resulted in a slight increase in CollAl expression. This is consistent with the data of previous studies showing that electromagnetic stimulation alters the expression of CollA1 and thus affects the osteoblastic proliferation phase $(20,34)$. The influence on differentiation was also displayed by a slight increase in $O C$ mRNA levels following stimulation with EMF. However, these findings were trends and did not reach a level of significance, whereas EMF + EF using the ASNIS-III s screw system resulted, not only in a significant enhancement of $O C$ and $A L P$ mRNA expression, but also in the increased expression of $R U N X-2$. These findings support those of previous studies showing that electrical stimulation promotes extracellular matrix maturation and mineralization $(35,36)$. Applied electric fields enhanced the production of important extracellular matrix proteins and further altered the gene regulation in terms of $R U N X-2$ expression, a key transcription factor associated with osteoblast differentiation (37). In conclusion, gene expression analyses showed a more pronounced effect of $\mathrm{EMF}+\mathrm{EF}$ on differentiation-associated markers compared to EMF alone. 
The results of the present study emphasise the capability of EMF + EF to enhance osteoblastic differentiation, and thus are consistent with those of our previous study that showed changes in collagen type 1 synthesis (12). However, the exposure of hOBs to electromagnetic fields with an additional electric field resulted in a significant and more pronounced elevation of synthesised collagen type 1 in a respective study, indicating the influence of scaffold composition on the response of cells to biophysical stimuli, as we and other study groups have proposed previously $(12,38)$. The agarosegel-scaffold used in the present study did not contain collagen fibres as opposed to the one used in our previous study. Collagen supports osteogenic differentiation by promoting extracellular matrix protein expression $(39,40)$. Additionally, the deformation of collagen fibres as a response to electromechanical coupling (inverse piezoelectric effect) may result in mechanically stimulated cell differentiation, thereby influencing bone remodelling $(12,22,41)$. The existence of the inverse piezoelectric effect in collagen fibres and its resulting deforming magnitude being sufficient to prompt mechanically induced stimulation was recently proven by our group through scanning X-ray diffraction experiments (11). Thus, the scaffold material may account for variances between our present and previous studies, indicating the beneficial but not the crucial effects of piezoelectric matrix components for the differentiation of hOBs following exposure to electromagnetic fields as previously demonstrated $(21,34,42)$. The distribution of the electric field generated by the ASNIS-III s screw derived from the numerical simulation study showed a considerable gradient within the scaffolds (12), limiting the intensity of EMF + EF in the periphery. Cells seeded in agarose are distributed equally throughout the gel-scaffold; therefore, more cells were subjected to a lower electric field in the outer edges, as opposed to cells seeded in the point-wise method used in our previous study which could also account for the differences between both studies. The positive stimulatory effects in the absence of extracellular matrix components prove the direct effect of electric fields on osteoblasts. The potential mechanisms involved include the release of calcium from intracellular stores and the activation of cytoskeletal calmodulin without the involvement of the inositol phosphate pathway or voltage gated calcium channels and phospholipase $\mathrm{A}_{2}$ activation as previously proposed (10).

In conclusion, in this study, we demonstrate the positive influence of EMF and EMF + EF on bone cell viability and differentiation even following short-term stimulation. Differentiation markers were significantly enhanced by stimulation with EMF + EF. These results emphasise the effectiveness of the clinically used implant system for promoting bone regeneration. Future studies regarding EMF + EF on hOBs with extended stimulation periods for the analysis of longterm effects and variation of parameters, including frequency, stimulation intervals and maximum electric field strength, may further optimise existing stimulation systems.

\section{Acknowledgements}

The authors would like to thank the German Research Foundation (DFG) for the financial support of this study via the grant BA 3347/2-2 and the GRK 1501 welisa.

\section{References}

1. Wolf JH: Julis Wolff and his 'law of bone remodeling'. Orthopade 24: 378-386, 1995 (In German).

2. Fukada E and Yasuda I: On the Piezoelectric Effect of Bone. J Phys Soc Jpn 12: 1158-1162, 1957.

3. Icaro Cornaglia A, Casasco M, Riva F, Farina A, Fassina L, Visai L and Casasco A: Stimulation of osteoblast growth by an electromagnetic field in a model of bone-like construct. Eur J Histochem 50: 199-204, 2006.

4. Soda A, Ikehara T, Kinouchi Y and Yoshizaki K: Effect of exposure to an extremely low frequency-electromagnetic field on the cellular collagen with respect to signaling pathways in osteoblast-like cells. J Med Invest 55: 267-278, 2008.

5. Hess R, Neubert H, Seifert A, Bierbaum S, Hart DA and Scharnweber D: A novel approach for in vitro studies applying electrical fields to cell cultures by transformer-like coupling. Cell Biochem Biophys 64: 223-232, 2012.

6. Hess R, Jaeschke A, Neubert H, Hintze V, Moeller S, Schnabelrauch M, Wiesmann HP, Hart DA and Scharnweber D: Synergistic effect of defined artificial extracellular matrices and pulsed electric fields on osteogenic differentiation of human MSCs. Biomaterials 33: 8975-8985, 2012.

7. Hronik-Tupaj M, Rice WL, Cronin-Golomb M, Kaplan DL and Georgakoudi I: Osteoblastic differentiation and stress response of human mesenchymal stem cells exposed to alternating current electric fields. Biomed Eng Online 10: 9, 2011.

8. Jansen JH, van der Jagt OP, Punt BJ, Verhaar JA, van Leeuwen JP, Weinans $\mathrm{H}$ and Jahr H: Stimulation of osteogenic differentiation in human osteoprogenitor cells by pulsed electromagnetic fields: An in vitro study. BMC Musculoskelet Disord 11: 188, 2010.

9. Saino E, Fassina L, Van Vlierberghe S, Avanzini MA, Dubruel P, Magenes G, Visai L and Benazzo F: Effects of electromagnetic stimulation on osteogenic differentiation of human mesenchymal stromal cells seeded onto gelatin cryogel. Int J Immunopathol Pharmacol 24 (Suppl 2): 1-6, 2011.

10. Brighton CT, Wang W, Seldes R, Zhang G and Pollack SR: Signal transduction in electrically stimulated bone cells. J Bone Joint Surg Am 83-A: 1514-1523, 2001.

11. Wieland DCF, Krywka C, Mick E, Willumeit-Römer R, Bader R and Kluess D: Investigation of the inverse piezoelectric effect of trabecular bone on a micrometer length scale using synchrotron radiation. Acta Biomater 25: 339-346, 2015.

12. Grunert PC, Jonitz-Heincke A, Su Y, Souffrant R, Hansmann D, Ewald H, Krüger A, Mittelmeier W and Bader R: Establishment of a novel in vitro test setup for electric and magnetic stimulation of human osteoblasts. Cell Biochem Biophys 70: 805-817, 2014.

13. Balint R, Cassidy NJ and Cartmell SH: Electrical stimulation: A novel tool for tissue engineering. Tissue Eng Part B Rev 19: 48-57, 2013.

14. Kang KS, Hong JM, Jeong YH, Seol YJ, Yong WJ, Rhie JW and Cho DW: Combined effect of three types of biophysical stimuli for bone regeneration. Tissue Eng Part A 20: 1767-1777, 2014.

15. Puricelli E, Dutra NB and Ponzoni D: Histological evaluation of the influence of magnetic field application in autogenous bone grafts in rats. Head Face Med 5: 1, 2009.

16. Goldstein C, Sprague S and Petrisor BA: Electrical stimulation for fracture healing: Current evidence. J Orthop Trauma 24 (Suppl 1): S62-S65, 2010.

17. Kuzyk PR and Schemitsch EH: The science of electrical stimulation therapy for fracture healing. Indian J Orthop 43: 127-131, 2009.

18. Niethard FU and Pfeil J: Duale Reihe Orthopädie. 5. Aufl. Thieme, Stuttgart, 2005.

19. Mittelmeier W, Lehner S, Kraus W, Matter HP, Gerdesmeyer L and Steinhauser E: BISS: Concept and biomechanical investigations of a new screw system for electromagnetically induced internal osteostimulation. Arch Orthop Trauma Surg 124: 86-91, 2004.

20. Fassina L, Visai L, Benazzo F, Benedetti L, Calligaro A, De Angelis MG, Farina A, Maliardi V and Magenes G: Effects of electromagnetic stimulation on calcified matrix production by SAOS-2 cells over a polyurethane porous scaffold. Tissue Eng 12: 1985-1999, 2006.

21. Griffin M, Sebastian A, Colthurst J and Bayat A: Enhancement of differentiation and mineralisation of osteoblast-like cells by degenerate electrical waveform in an in vitro electrical stimulation model compared to capacitive coupling. PLoS One 8: e72978, 2013. 
22. Gillespie PG and Walker RG: Molecular basis of mechanosensory transduction. Nature 413: 194-202, 2001.

23. Lochner K, Fritsche A, Jonitz A, Hansmann D, Mueller P, Mueller-Hilke B and Bader R: The potential role of human osteoblasts for periprosthetic osteolysis following exposure to wear particles. Int J Mol Med 28: 1055-1063, 2011.

24. Livak KJ and Schmittgen TD: Analysis of relative gene expression data using real-time quantitative PCR and the 2(-Delta Delta C(T)) Method. Methods 25: 402-408, 2001.

25. Dimitriou R, Jones E, McGonagle D and Giannoudis PV: Bone regeneration: Current concepts and future directions. BMC Med 9: 66, 2011.

26. Watanabe J,Kashii M,Hirao M,OkaK, SugamotoK, Yoshikawa H and Akashi M: Quick-forming hydroxyapatite/agarose gel composites induce bone regeneration. J Biomed Mater Res A 83: 845-852, 2007.

27. Hanazaki Y, Ito D, Furusawa K, Fukui A and Sasaki N: Change in the viscoelastic properties of agarose gel by HAp precipitation by osteoblasts cultured in an agarose gel matrix. J Biorheol 1-2: 21-28, 2013.

28. Tabata M, Shimoda T, Sugihara K, Ogomi D, Ohgushi H and Akashi M: Apatite formed on/in agarose gel as a bone-grafting material in the treatment of periodontal infrabony defect. J Biomed Mater Res B Appl Biomater 75: 378-386, 2005.

29. Ji J, Sun W, Wang W, Munyombwe T and Yang XB: The effect of mechanical loading on osteogenesis of human dental pulp stromal cells in a novel in vitro model. Cell Tissue Res 358: 123-133, 2014.

30. Bordji K, Jouzeau JY, Mainard D, Payan E, Netter P, Rie KT, Stucky T and Hage-Ali M: Cytocompatibility of Ti-6Al-4V and Ti-5Al-2.5Fe alloys according to three surface treatments, using human fibroblasts and osteoblasts. Biomaterials 17: 929-940, 1996.

31. Eisenbarth E, Velten D, Müller M, Thull R and Breme J: Biocompatibility of $\beta$-stabilizing elements of titanium alloys. Biomaterials 25: 5705-5713,2004.

32. Long M and Rack HJ: Titanium alloys in total joint replacement - a materials science perspective. Biomaterials 19: 1621-1639, 1998.

33. Fassina L, Visai L, De Angelis MG, Benazzo F and Magenes G: Surface modification of a porous polyurethane through a culture of human osteoblasts and an electromagnetic bioreactor. Technol Health Care 15: 33-45, 2007.
34. Lohmann CH, Schwartz Z, Liu Y, Guerkov H, Dean DD, Simon B and Boyan BD: Pulsed electromagnetic field stimulation of MG63 osteoblast-like cells affects differentiation and local factor production. J Orthop Res 18: 637-646, 2000.

35. Meng S, Rouabhia $M$ and Zhang Z: Electrical stimulation modulates osteoblast proliferation and bone protein production through heparin-bioactivated conductive scaffolds. Bioelectromagnetics 34: 189-199, 2013.

36. Meng S, Zhang Z and Rouabhia M: Accelerated osteoblast mineralization on a conductive substrate by multiple electrical stimulation. J Bone Miner Metab 29: 535-544, 2011.

37. Vimalraj S, Arumugam B, Miranda PJ and Selvamurugan N: Runx2: Structure, function, and phosphorylation in osteoblast differentiation. Int J Biol Macromol 78: 202-208, 2015.

38. Dubey AK, Gupta SD and Basu B: Optimization of electrical stimulation parameters for enhanced cell proliferation on biomaterial surfaces. J Biomed Mater Res B Appl Biomater 98: 18-29, 2011.

39. Salasznyk RM, Klees RF, Hughlock MK and Plopper GE: ERK signaling pathways regulate the osteogenic differentiation of human mesenchymal stem cells on collagen I and vitronectin. Cell Commun Adhes 11: 137-153, 2004.

40. Salasznyk RM, Williams WA, Boskey A, Batorsky A and Plopper GE: Adhesion to vitronectin and collagen I promotes osteogenic differentiation of human mesenchymal stem cells. J Biomed Biotechnol 2004: 24-34, 2004.

41. Bistolfi F: Evidence of interlinks between bioelectromagnetics and biomechanics: From biophysics to medical physics. Phys Med 22: 71-95, 2006.

42. Kim IS, Song JK, Zhang YL, Lee TH, Cho TH, Song YM, Kim K, Kim SJ and Hwang SJ: Biphasic electric current stimulates proliferation and induces VEGF production in osteoblasts. Biochim Biophys Acta 1763: 907-916, 2006. 